\title{
General Fatigue Dimension
}

National Cancer Institute

\section{Source}

National Cancer Institute. General Fatigue Dimension. NCI Thesaurus. Code C132282.

A dimension of general fatigue in the Multidimensional Fatigue Inventory. 